Inder K. Rana, Mathematics Department, Indian Institute of Technology, Powai, Bombay - 400 076, India, email: IKR@@ganit.math.iitb.ernet.in

\title{
ON APPROXIMATE UNSMOOTHING OF FUNCTIONS
}

\begin{abstract}
The smoothing $T_{a} f$, for $a>0$, of a locally-integrable function $f$ : $\mathbb{R} \longrightarrow \mathbb{R}$ is defined by

$$
\left(T_{a} f\right)(x):=\frac{1}{2 a} \int_{-a}^{+a} f(x+y) d y, \quad x \in \mathbb{R} .
$$

For a given $g: \mathbb{R} \longrightarrow \mathbb{R}$, any solution $f$ of the equation $T_{a} f=g$ is called an unsmoothing of $g$. In this note we analyse the problem of constructing a function $\tilde{f}: \mathbb{R} \longrightarrow \mathbb{R}$ such that $\left(T_{a} \tilde{f}\right)\left(x_{i}\right)=g\left(x_{i}\right)$ for a given set of points $x_{1}, x_{2}, \ldots, x_{n} \in \mathbb{R}$. We give an iterative process of constructing such an $\tilde{f}$ under the assumption $f \in L_{2}(\mathbb{R})$.
\end{abstract}

\section{Introduction}

Let $L_{l o c}(\mathbb{R})$ denote the space of all locally-integrable functions on the real line and for $a>0, f \in L_{l o c}(\mathbb{R})$, let

$$
\left(T_{a} f\right)(x):=\frac{1}{2 a} \int_{-a}^{+a} f(x+y) d y, x \in \mathbb{R} .
$$

The function $T_{a} f$ is called the smoothing of $f$ and $T_{a}$ is called the smoothing operator. In [2], the range and kernel of the smoothing operator were discussed and a right-inverse for $T_{a}$ was constructed which preserved the differentiability properties optimally. In practical problems, $T_{a} f$ represents the smoothing (moving average or sliding mean) of the raw data $f$. The problem of constructing some function $f$ such that $T_{a} f=g, g$ given, is called the

Mathematical Reviews subject classification: Primary: 45E10 Secondary: 45L99

Received by the editors December 8, 1994 
unsmoothing problem. In the case when $f$ is an integrable function with compact support, reconstruction formulas using two-sided Laplace transform were obtained by Van der Pol in [3].

We consider the following situation : let a function $g$ be the smoothing of some function $f$, i.e., $T_{a} f=g$. One knows $g$ at only a finite number of points, i.e., one knows the values $g\left(x_{i}\right)=c_{i}$, for some $x_{1}, x_{2}, \ldots, x_{n} \in \mathbb{R}$. Of course, one cannot hope to recover the function $f$ exactly from this data. However, it is meaningful to ask the question: Can one find some $\tilde{f}$ such that $\left(T_{a} \tilde{f}\right)=c_{i}, 1 \leq i \leq n$ ? Since, in practical situations, the raw data $f(x)$ is a bounded function (at least on finite time intervals), it is not unreasonable to assume that there exists some $f$ which is bounded and has compact support such that $\left(T_{a} f\right)\left(x_{i}\right)=c_{i}, 1 \leq i \leq n$. So, we may assume that there exists some $f \in L_{2}(\mathbb{R})$ such that $\left(T_{a} f\right)\left(x_{i}\right)=c_{i}, 1 \leq i \leq n$. The problem is to find some $\tilde{f} \in L_{2}(\mathbb{R})$ such that $\left(T_{a} \tilde{f}\right)\left(x_{i}\right)=c_{i}, 1 \leq i \leq n$. We call this the approximate unsmoothing problem.

\section{Construction of 'Approximate Unsmoothing'}

We are given $x_{1}, x_{2}, \ldots, x_{n} \in \mathbb{R}$ and $c_{1}, c_{2}, \ldots, c_{n} \in \mathbb{R}$. We also have the knowledge that there exists some $f \in L_{2}(\mathbb{R})$ such that $\left(T_{a} f\right)\left(x_{i}\right)=c_{i}, 1 \leq i \leq$ $n$. We want to construct some $\tilde{f} \in L_{2}(\mathbb{R})$ such that $\left(T_{a} \tilde{f}\right)\left(x_{i}\right)=c_{i}, 1 \leq i \leq n$. In case the intervals $\left[x_{i}-a, x_{i}+a\right], 1 \leq i \leq n$ are pairwise disjoint, an obvious choice for $\tilde{f}$ is given by

$$
\tilde{f}=\sum_{i=1}^{n} c_{i} \chi_{\left[x_{i}-a, x_{i}+a\right]}
$$

It is easy to see that $\left(T_{a} \tilde{f}\right)\left(x_{i}\right)=c_{i}, 1 \leq i \leq n$. In the general case, we proceed as follows: We choose arbitrary functions $\phi_{i} \in L_{2}(\mathbb{R})$, such that $\left(T_{a} \phi_{i}\right)\left(x_{i}\right)=1,1 \leq i \leq n$ (for example $\phi_{i}=\chi_{\left[x_{i}-a, x_{i}+a\right]}$ ). Let $\langle.,$.$\rangle denote$ the inner-product on $L_{2}(\mathbb{R})$ and

$$
N_{i}=\left\{g \in L_{2}(\mathbb{R}) \mid\left\langle g, \chi_{\left[x_{i}-a, x_{i}+a\right]}\right\rangle=0\right\}, 1 \leq i \leq n .
$$

Then each $N_{i}$ is a closed subspace of $L_{2}(\mathbb{R})$ of co-dimension one. Let

$$
Q_{i}: L_{2}(\mathbb{R}) \longrightarrow N_{i}
$$

denote the orthogonal projection onto $N_{i}$. Let $P_{i}: L_{2}(\mathbb{R}) \longrightarrow L_{2}(\mathbb{R})$ be defined by

$$
P_{i}(g):=Q_{i}(g)+\left(I-Q_{i}\right)(f), \quad \forall g \in L_{2}(\mathbb{R}) .
$$


We note that $\left(I-Q_{i}\right)(f) \in \operatorname{span}\left\{\phi_{i}\right\}$ and hence

$$
\left(I-Q_{i}\right)(f)=\alpha_{i} \phi_{i}, \quad \text { for some } \alpha_{i} \in \mathbb{R} .
$$

In fact,

$$
\begin{aligned}
\alpha_{i} & =\alpha_{i}\left[\left(T_{a} \phi_{i}\right)\left(x_{i}\right)\right]=\left[T_{a}\left(\alpha_{i} \phi_{i}\right)\right]\left(x_{i}\right) \\
& =\left[T_{a}\left(\left(I-Q_{i}\right)(f)\right)\right]\left(x_{i}\right)=\left(T_{a} f\right)\left(x_{i}\right)-\left[\left(T_{a} Q_{i}\right)(f)\right]\left(x_{i}\right) \\
& =c_{i}-\left\langle Q_{i}(f), \chi_{\left[x_{i}-a, x_{i}+a\right]}\right\rangle=c_{i} .
\end{aligned}
$$

Thus,

$$
P_{i}(g)=Q_{i}(g)+c_{i} \phi_{i}
$$

Hence, the operators $Q_{i}$ and $P_{i}, 1 \leq i \leq n$ are completely known once the functions $\phi_{i}$ 's are chosen. For further arguments, we need the following :

Theorem 2.1 Let $\mathcal{H}$ be a Hilbert space and $Q_{i}$ be the orthogonal projection onto a closed subspace $N_{i} \subset \mathcal{H}, 1 \leq i \leq n$. Let $N_{0}=\bigcap_{i=1}^{n} N_{i}$ and $Q_{0}$ be the orthogonal projection onto $N_{0}$. Let $Q=Q_{n} Q_{n-1} \ldots Q_{1}$. Then $Q^{m}(g) \rightarrow$ $Q_{0}(g)$ as $m \rightarrow \infty$ for every $g \in \mathcal{H}$.

Proof. We refer to [1].

Lemma 2.2 Let $Q_{i}, P_{i}, 1 \leq i \leq n$ be as constructed in (1) and (2). Let $Q_{0}$ denote the orthogonal projection on the subspace $N_{0}=\cap_{i=1}^{n} N_{i}$ and let $Q=$ $Q_{n} Q_{n-1} \ldots Q_{1}, P=P_{n} P_{n-1} \ldots P_{1}$ and $P_{0}(g)=Q_{0}(g-f)+f, g \in L_{2}(\mathbb{R})$. Then $\lim _{m \rightarrow \infty} P^{m}(g)=P_{0}(g) \forall g \in L_{2}(\mathbb{R})$.

Proof. We first show that $\forall m$ and $\forall g \in L_{2}(\mathbb{R}), P^{m}(g)=Q^{m}(g-f)+f$. For $m=1$ and $g \in L_{2}(\mathbb{R})$,

$$
\begin{aligned}
P(g)= & P_{n}\left(P_{n-1} \ldots\left(P_{1}(g)\right)\right) \\
= & Q_{n}\left(P_{n-1} \ldots\left(P_{1}(g)\right)\right)+\left(I-Q_{n}\right)(f) \\
= & Q_{n}\left[Q_{n-1}\left(P_{n-2} \ldots\left(P_{1}(g)\right)\right)+\left(I-Q_{n-1}\right)(f)\right]+\left(I-Q_{n}\right)(f) \\
= & Q_{n}\left[Q_{n-1}\left(P_{n-2} \ldots\left(P_{1}(g)\right)\right)\right]+\left(I-Q_{n} Q_{n-1}\right)(f) \\
& \ldots \ldots . . \\
= & Q_{n}\left(Q_{n-1} \ldots\left(Q_{1}(g)\right)\right)+\left(I-Q_{n} Q_{n-1} \ldots Q_{1}\right)(f) \\
= & Q(g-f)+f .
\end{aligned}
$$


Suppose the claim is true for each $m \leq k-1$. Then $\forall g \in L_{2}(\mathbb{R})$

$$
\begin{aligned}
P^{k}(g) & =P^{k-1}(P(g))=Q^{k-1}(P(g)-f)+f \\
& =Q^{k-1}(Q(g-f))+f=Q^{k}(g-f)+f .
\end{aligned}
$$

Thus, by induction, $\forall m \geq 1$ and $g \in L_{2}(\mathbb{R})$

$$
P^{m}(g)=Q^{m}(g-f)+f .
$$

By Theorem 2.1, $Q^{m}(g-f) \longrightarrow Q_{0}(g-f)$, where $Q_{0}$ is the orthogonal projection onto $\cap_{i=1}^{n} N_{j}$. Hence $P^{m}(g) \longrightarrow Q_{0}(g-f)+f=P_{0}(g) \forall g \in L_{2}(\mathbb{R})$.

Theorem 2.3 Let $g_{0} \in L_{2}(\mathbb{R})$ be arbitrary and let $P$ be as in Lemma 2.2. Then $\left\{P^{m}\left(g_{0}\right)\right\}_{m \geq 1}$ is convergent and if $\tilde{f}:=\lim _{m \rightarrow \infty} P^{m}\left(g_{0}\right)$, then

$$
\left(T_{a} \tilde{f}\right)\left(x_{i}\right)=c_{i}, 1 \leq i \leq n .
$$

Proof. By Lemma 2.2, $\left\{P^{m}\left(g_{0}\right)\right\}_{m \geq 1}$ converges to $Q_{0}\left(g_{0}-f\right)+f$. Thus, if $\tilde{f}:=Q_{0}\left(g_{0}-f\right)+f$, then $\tilde{f}-f=Q_{0}\left(g_{0}-f\right) \in N_{0}$. Hence $\forall i=1,2, \ldots, n$,

$$
0=\left\langle\tilde{f}-f, \chi_{\left[x_{i}-a, x_{i}+a\right]}\right\rangle=\int_{x_{i}-a}^{x_{i}+a}(\tilde{f}-f)(x) d x=2 a\left(T_{a}(\tilde{f}-f)\left(x_{i}\right) .\right.
$$

Thus $T_{a}\left(\tilde{f}\left(x_{i}\right)\right)=T_{a}(f)\left(x_{i}\right)=c_{i} \forall 1 \leq i \leq n$.

This completes the proof of the theorem.

Note 2.4 It is easy to see that the approximate unsmoothing can also be constructed in the case $\mathbb{R}$ is replaced by $\mathbb{R}^{n}$ and intervals $\left[x_{i}-a, x_{i}+a\right]$ are replaced by bounded subsets of $\mathbb{R}^{n}$ of positive Lebesgue measure.

Acknowledgement : Author would like to thank Prof. A. Sitaram for his suggestions.

\section{References}

[1] I. Amemiya and T. Ando. Convergence of random products of contractions in Hilbert spaces, Acta Sci. Math. (Szeged) 26 (1965), 239-244.

[2] E. Novakand Inder K. Rana, On the unsmoothing of functions on the real line, Proc. Nede. Acad. Sci. Series A, 89 (1986), 201-207.

[3] B. Van der Pol, Smoothing and Unsmoothing, contained in Probability and Related Topics in Physical Sciences by M. Kac, Interscience, New York, 1959. 